\title{
Measurements of Ionization Cross Sections by Molecular Beam Experiments: Information Content on the Imaginary Part of the Optical Potential
}

\author{
Stefano Falcinelli, ${ }^{* \dagger}{ }^{\dagger}$ Marzio Rosi, ${ }^{\dagger}$ Fernando Pirani, $^{\ddagger}$ Domenico Stranges, ${ }^{\S}$ and Franco Vecchiocattivi ${ }^{\dagger}$ \\ ${ }^{\dagger}$ Department of Civil and Environmental Engineering, University of Perugia, Via G. Duranti 93, 06125 Perugia, Italy \\ ${ }^{\ddagger}$ Department of Chemistry, Biology and Biotechnologies, University of Perugia, Via Elce di Sotto, 8, 06123 Perugia, Italy \\ ${ }^{\S}$ Department of Chemistry, University of Rome "La Sapienza”, 00185 Rome, Italy
}

ABSTRACT: In this work, we present and analyze in detail new and recent ionization cross section and mass spectrum determinations, collected in the case of $\mathrm{He}^{*}, \mathrm{Ne}^{*}-\mathrm{H}_{2} \mathrm{O},-\mathrm{H}_{2} \mathrm{~S}$, and $-\mathrm{NH}_{3}$ ionizing collisions. These sets of data, obtained under the same experimental conditions, are relevant to identify differences in the autoionization stereodynamics of the three hydrogenated molecules and on the selective role of the imaginary part of the optical potential. We demonstrate that in these autoionization processes hydrogen and halogen bonds are competing because they are controlling both real and imaginary components of the optical potential that drives the complete reaction dynamics. In particular, we found that both components critically depend on the angular and radial approach between the reagent partners in determining the collision dynamics.

\section{A GENERAL INTRODUCTION}

The collisional approach between reagents is crucial in determining the stereodynamics of reactions occurring in gaseous and condensed phase. In particular, the collision between an excited atomic species (for example, a rare gas metastable atom) and an atomic or molecular target is relevant in cold and rarefied plasma environments, such as planetary ionospheres and the interstellar medium where the reaction occurs in a single collision condition without the average overall possible orientations, and then it maintains memory of the selectivity of each collision event. This selectivity emerges also under thermal collisions, which is typical of the tropospheric chemistry.

Because of the lacking of detailed information on the microscopic dynamics, the orientation of reagents species is often neglected in studies aimed to evaluate physical and chemical properties depending on the reagent collision energy, such as rate constants and cross sections. In this paper, we report on the dependence of measured absolute total ionization cross sections as a function of the collision energy. The use of analytical formulation of the potential energy surfaces (PESs) allows us to extract crucial information on the stereodynamics of the autoionization processes of relevance in several fields, because the dependence of these phenomena on relative orientation of involved partners.

The elementary reactions of interest can be schematized as follows

$$
\begin{aligned}
& \mathrm{Rg}^{*}+\mathrm{M} \rightarrow[\mathrm{Rg} . . . \mathrm{M}]^{*} \rightarrow[\mathrm{Rg} . . . \mathrm{M}]^{+}+\mathrm{e}^{-} \\
& {[\mathrm{Rg} . . \mathrm{M}]^{+} \rightarrow \text { neutral and ionic products }}
\end{aligned}
$$

where $\mathrm{Rg}$ is a rare gas atom, $\mathrm{M}$ is an atomic or molecular target, $[\mathrm{Rg} . . \mathrm{M}] *$ represents the intermediate autoionizing collisional complex, and $[\mathrm{Rg} . . . \mathrm{M}]^{+}$is the intermediate transient ionic species formed by the electron ejection. The $[\mathrm{Rg} . . . \mathrm{M}] *$ complex has a lifetime so short $\left(\sim 10^{-15} \mathrm{~s}\right)$ that it can be considered as very close to the true "transition state" of the process. $^{1}$

These reactions are commonly named as "Penning ionization" processes, after the early observation in 1927 by F. M. Penning. ${ }^{2}$ They occur when the ionization potential of the target particle is lower than the excitation energy of the metastable species. It is of relevance to remember that rare gas atoms excited to their first electronic level are metastable with a lifetime long enough to allow them to survive longer than the collision time and make them able to ionize upon collision many atomic and molecular targets. Moreover, metastable rare gas atoms have a large excitation energy, and in particular $\mathrm{He}^{*}\left(2^{3} \mathrm{~S}_{0},{ }^{1} \mathrm{~S}_{1}\right)$ and $\mathrm{Ne} *\left({ }^{3} \mathrm{P}_{2},{ }^{3} \mathrm{P}_{0}\right)$ have enough energy to ionize almost all molecular species.

An interesting characteristic of these processes is that they exhibit a rate constant comparable with those of many bimolecular reactions of atmospheric interest, such as those involving $\mathrm{O}$ atoms (in the metastable ${ }^{1} \mathrm{D}$ state) and/or other open shell species, such as $\mathrm{Cl}, \mathrm{Br}$, or $\mathrm{OH}^{3,4}$ This means that collisional autoionizations are in general fast reactions, which play an important role in different topics of the research in fundamental and applied physical chemistry, for example, the characterization of thermal plasmas, electrical discharges, and the production of laser systems. ${ }^{5,6}$ The possible role of

Special Issue: Piergiorgio Casavecchia and Antonio Lagana Festschrift

Received: January 25, 2016

Revised: March 2, 2016 
collisional autoionization reactions in the chemistry of planetary atmospheres has been recently discussed in some recent papers. ${ }^{7,8}$ For instance, free ionic species are extremely important for the transmission of radio and satellite signals. In fact, due to the presence of high amounts of ions and free electrons the ionosphere plays an important role in the transmission of electromagnetic waves, especially $\mathrm{HF}$ radio and satellite waves. ${ }^{8}$ In addition, the role of metastable rare gas atoms in Earth's atmospheric reactions is of interest for other reasons: first, the quantity of rare gas atoms in the atmosphere is not negligible and moreover, recent studies have shown that the exosphere of our planet is rich in $\mathrm{He}^{*}\left(2^{1} \mathrm{~S}_{0}\right)$ metastable atoms. $^{9-12}$ Despite their importance, relevant details on the microscopic dynamics of such reactions is presently lacking and this paper aims to contribute to partially fill this gap, through a combined analysis of new and recent experimental data that cast light on the features of the passage from reagents to product states.

From a fundamental point of view, it has to be noted that the collision dynamics is directly controlled by the intermolecular potential driving approach and removing of reactants and products, respectively. In the case of autoionization processes, the total intermolecular potential is determined by the combination of a real component, $V$, which drives the system in the entrance and exit channels, and an imaginary part, $\Gamma$, defining the ionization probability, because of triggering the passage from reagents to products. Such intermolecular potential, according to the so-called "optical potential model", well described in some review papers, ${ }^{13,14}$ is characterized by a compact analytical formulation reported in eq 2

$$
W(R, \theta, \phi)=V(R, \theta, \phi)-\frac{i}{2} \Gamma(\mathrm{R}, \theta, \phi)
$$

where $R$ represents the distance of the metastable atom from the center of mass of the target molecule, whereas $\theta$ and $\phi$ are the polar angles defining the relative orientation. ${ }^{4}$

As mentioned above, the case of the collisional autoionization of hydrogenated molecules is of great interest for both interstellar medium processes and planetary phenomena. In fact, water, ammonia, and hydrogen sulfide have been recently found in a considerable amount in such environments as the Jovian planets and Titan. ${ }^{15}$ As stressed above, we have addressed the present study on the characterization of the stereodynamics of the autoionization of such molecules.

\section{THE SUBJECT OF THE PRESENT WORK}

In this paper, the focus is on the measure of total and partial ionization cross sections for autoionization of water, ammonia, and hydrogen sulfide by metastable helium and neon atoms. Their combined analysis, which also considers the emitted electron energy spectra, can provide useful information about the microscopic dynamics of these reactions. In particular, as it will be discussed in Section 3, by the use of a semiclassical treatment of the collision dynamics, together with potential energy surfaces whose formulation is given in an analytical form and as a function of fundamental physical properties of the reactants, we are able to calculate and analyze the total ionization cross section as a function of the collision energy for different approaching angles between $\mathrm{Rg}^{*}$ (with $\mathrm{Rg}=\mathrm{He}, \mathrm{Ne}$ ) and simple hydrogenated molecular reagents $\left(\mathrm{H}_{2} \mathrm{O}, \mathrm{H}_{2} \mathrm{~S}\right.$, and $\mathrm{NH}_{3}$ ). Such an analysis appears suitable for quantitative molecular dynamics calculations allowing one to control the effect of the angular approach between the reagent particles.
Therefore, it becomes possible to perform a detailed stereodynamics study of Penning ionization reactions, considering in the "optical potential model" formulation of eq 2 the explicit dependence on the approaching angle between reagents. Both the semiclassical treatment and the potential energy surface formulation have been developed in our laboratory and the obtained final results show the evidence that the autoionization process is controlled by the competition between the formation of a hydrogen bondlike complex $\left[\mathrm{Rg}-\mathrm{H}_{n} \mathrm{X}\right] *$ (where $\mathrm{X}=\mathrm{O}, \mathrm{S}$, or $\mathrm{N}$ and $n=2,3$, respectively) when the $\mathrm{Rg}^{*}$ atom approaches the molecule toward the $\mathrm{H}$-end side, and the formation of a halogen bond like complex $\left[\mathrm{Rg}-\mathrm{XH}_{n}\right]^{*}$ when the approach occurs toward the lone pair molecular side. ${ }^{16,17}$ In the latter configuration the ionization is stimulated by a strong attractive halogen bond interaction and by a pronounced electron exchange probability between a filled orbital of the molecule and the inner shell hole of the ionic core of the $\mathrm{Rg}^{*}$ atom. Both combined effects point out that the reactive events mostly occur when a halogen bondlike complex $\left[\mathrm{Rg}-\mathrm{XH}_{n}\right] *$ is formed and molecular orientation effects become evident in the ionization cross section measurements at low collision energy range.

It has to be stressed that the present work clearly indicates that in the case of autoionization processes of hydrogenated molecules both real and imaginary components are depending on the balance of some leading interaction terms responsible of the formation of an intermolecular hydrogen and halogen bond.

In our recent papers, ${ }^{17-20}$ we have basically exploited a combined analysis of the Penning ionization electron spectra, measured under the same conditions, which represent a sort of a transition state spectroscopy. ${ }^{1}$ Such an analysis, focused on the shift of the measured peaks ${ }^{21}$ with respect to the position expected without the intermolecular interaction, represented a detailed probe of strength and angular dependence of the real part of the involved interactions. This was possible because such a component affects the energetics and structure of the transition state.

In this paper, we present new measurements of ionization cross sections, to be compared with data recently published, in order to perform an internally consistent comparison, since they represent observables measured under the same experimental conditions. In particular, new unpublished data are presented about $\mathrm{Ne}^{*}-\mathrm{H}_{2} \mathrm{~S}$ and $\mathrm{Ne}^{*}-\mathrm{H}_{2} \mathrm{O}$ systems, where in the latter case additional cross-section data are extending the energy range previously investigated. ${ }^{6}$ Their combined analysis, together with those recently reported ${ }^{18}$ for $\mathrm{Ne}^{*}-\mathrm{NH}_{3}$ allows the emphasis of new aspects of the stereodynamics of these autoionization reactions, as discussed below.

The observed experimental findings are indeed of great relevance to properly assess the role of the imaginary component of the optical model, reported in eq 2, which triggers the reaction probability. It must to be stressed that in previous analyses the imaginary component was assumed to be only radial dependent and exponentially decreasing with the intermolecular distance. The comparative analysis of value and energy dependence of the measured cross sections is crucial to emphasize for the first time the selective role of strength, radial, and angular dependence of the imaginary component.

In Section 3, the experimental methodology is summarized in order to specify the used conditions. In Section 4, the experimental data are presented and discussed in a comparative way in order to emphasize the information content of the absolute cross section value and of the different dependence on 
the collision energy. A discussion and some conclusions are then given in Sections 4 and 5.

\section{MATERIALS AND METHODS}

3.1. Materials. All used reagent gases are from commercial cylinders at room temperature and were supplied to a needle effusive beam source. The $\mathrm{He}, \mathrm{Ne}, \mathrm{H}_{2} \mathrm{~S}$, and $\mathrm{NH}_{3}$ gases have a 99.99\% nominal purity and were used without any further treatment. The $\mathrm{H}_{2} \mathrm{O}$ molecular beam reagent was prepared by effusion of ultrapure water vapor from a glass microcapillary array kept at $30 \pm 1{ }^{\circ} \mathrm{C}$ by using a thermostatic system.

3.2. Experimental Method. The experimental setup, consisting in a crossed molecular beam apparatus (three vacuum chambers that are differentially pumped at a pressure of about $10^{-7} \mathrm{mbar}$ ) is described in detail in some previous works. ${ }^{5,22,23}$ A scheme of such an apparatus is reported in Figure 1, where it can be seen that a molecular beam of excited metastable rare gas atoms is crossing at right angles a second beam of molecules (to be ionized).

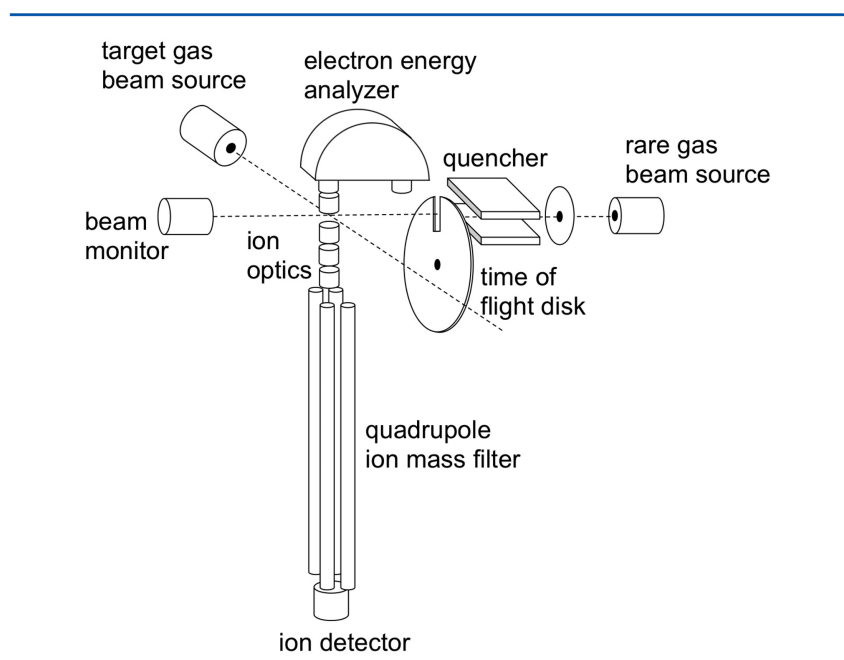

Figure 1. A schematic sketch of the experimental setup used for the crossed beam measurement of the ionization cross sections.

Electrons produced in the reaction are extracted and analyzed by an appropriate electron energy selector, while product ions are sent into a quadrupole mass spectrometer, where they are mass analyzed and detected. The metastable rare gas beam crosses at right angles an effusive beam of $\mathrm{H}_{2} \mathrm{O}$, $\mathrm{H}_{2} \mathrm{~S}$, or $\mathrm{NH}_{3}$ target molecules.

In the mass spectrometric (MS) determinations, in order to measure the total ionization cross section in a wide range of collision velocities the neon beam is produced by two sources, which can be used alternately. The first one is a standard effusive source, maintained at room temperature and coupled with a $150 \mathrm{eV}$ electron bombardment device, while the second one is a microwave discharge beam source operating with pure neon at a pressure of $\sim 10^{-3} \mathrm{~atm}$. The charged particles and the high-Rydberg-state atoms that can be also produced with these kinds of sources are removed from the beam by applying an electric field of about $2 \mathrm{kV} \mathrm{cm}$. It has to be noted that together with metastable atoms, the discharge source produces a large amount of $\mathrm{He}(\mathrm{I})$ and $\mathrm{Ne}(\mathrm{I})$ photons (depending on the used rare gas), having almost the same energy content (21.22 $\mathrm{eV}$ and, mainly, $16.84 \mathrm{eV}$, respectively) of the corresponding excited metastable atoms. These energy values allowed comparative studies of Penning ionization and photoionization. $^{5,18}$

For the $\mathrm{Ne}^{*}-\mathrm{H}_{2} \mathrm{O}, \mathrm{H}_{2} \mathrm{~S}$, and $\mathrm{NH}_{3}$ total ionization crosssection determinations, the $\mathrm{Ne}^{*}$ atom velocity is analyzed by a time-of-flight (TOF) technique; the beam is pulsed by a rotating slotted disk and the metastable atoms are counted, using a multiscaler, as a function of the delay time from the beam opening. In such a way, the velocity dependence of the cross section is obtained. Time delay spectra of the metastable atom arrival at the collision zone are recorded, as well as the time spectra of the product ion intensity. Then the relative cross section, $\sigma$, as a function of the collision energy, $E$, are obtained for a given delay time, $\tau$, according to eq 3

$$
\sigma(E)=\frac{I^{+}(\tau) v_{1}}{I^{*}(\tau) g}
$$

where $I^{+}$and $I^{*}$ are the intensities of the product ions and of the metastable atoms, respectively, $v_{1}$ is the laboratory velocity of the $\mathrm{Ne}^{*}$, and $g$ is the average relative collision velocity. By the TOF technique, the separation of photo ions and Penning ions is very easy with the former being detected at practically zero delay time. Absolute values of the total ionization cross section for different species can be obtained by the measurement of relative ion intensities in the same conditions of both metastable atom and target gas densities in the crossing volume. This allows the various systems to be put on a relative scale that can be then normalized by reference to a known cross section such as in the present case the $\mathrm{Ne}^{*}-\mathrm{Ar}$ absolute total ionization cross-section by West et al., ${ }^{24}$ which is known to be quite reliable. ${ }^{25}$

\section{RESULTS AND DISCUSSION}

In this section, experimental mass spectrometric and crosssection determinations, concerning production and characterization of simple ionic species produced by gas phase autoionization collisions of hydrogenated molecules $\left(\mathrm{H}_{2} \mathrm{O}\right.$, $\mathrm{H}_{2} \mathrm{~S}$, and $\mathrm{NH}_{3}$ ) with $\mathrm{He}^{*}$ and $\mathrm{Ne}^{*}$ metastable atoms, are reported. All experiments have been performed in the thermal collision energy range. The results are presented and discussed in a comparative way because they were measured under the same conditions. In the mass spectrometric determinations, we have extended the investigated collision energy range in respect to previous published data concerning $\mathrm{Ne}^{*}+\mathrm{H}_{2} \mathrm{O}$ system, ${ }^{6}$ while for $\mathrm{Ne}^{*}-\mathrm{H}_{2} \mathrm{~S}$ these data are new results. Further, for $\mathrm{Ne}^{*}-\mathrm{H}_{2} \mathrm{O},-\mathrm{H}_{2} \mathrm{~S}$, and $-\mathrm{NH}_{3}$ systems, the mass spectrometric study allowed us to determine also the absolute values of total ion production cross sections. In particular, the cross sections are put in an absolute scale by using the procedure described in Section 2, accordingly to eq 3. For the three systems, a comparison of the absolute values, evaluated at the average collision energy in the probed range $(\sim 70 \mathrm{meV})$, is given in Table 1. The estimated uncertainty of this kind of

Table 1. Absolute Total Ionization Cross Section Values for Autoionizing Collisions of Hydrogenated Molecules by $\mathrm{Ne}^{*}$ Metastable Atoms Measured at a Relative Collision Energy of $70 \mathrm{meV}$, Following the Procedure Described in Section 3

$\begin{array}{cc}\text { autoionizing system } & \text { total ionization cross section }\left(\AA^{2}\right) \\ \mathrm{Ne}^{*}-\mathrm{H}_{2} \mathrm{O} & 47.5 \pm 2.7 \\ \mathrm{Ne}^{*}-\mathrm{H}_{2} \mathrm{~S} & 42.4 \pm 2.4 \\ \mathrm{Ne}^{*}-\mathrm{NH}_{3} & 52.3 \pm 3.0\end{array}$




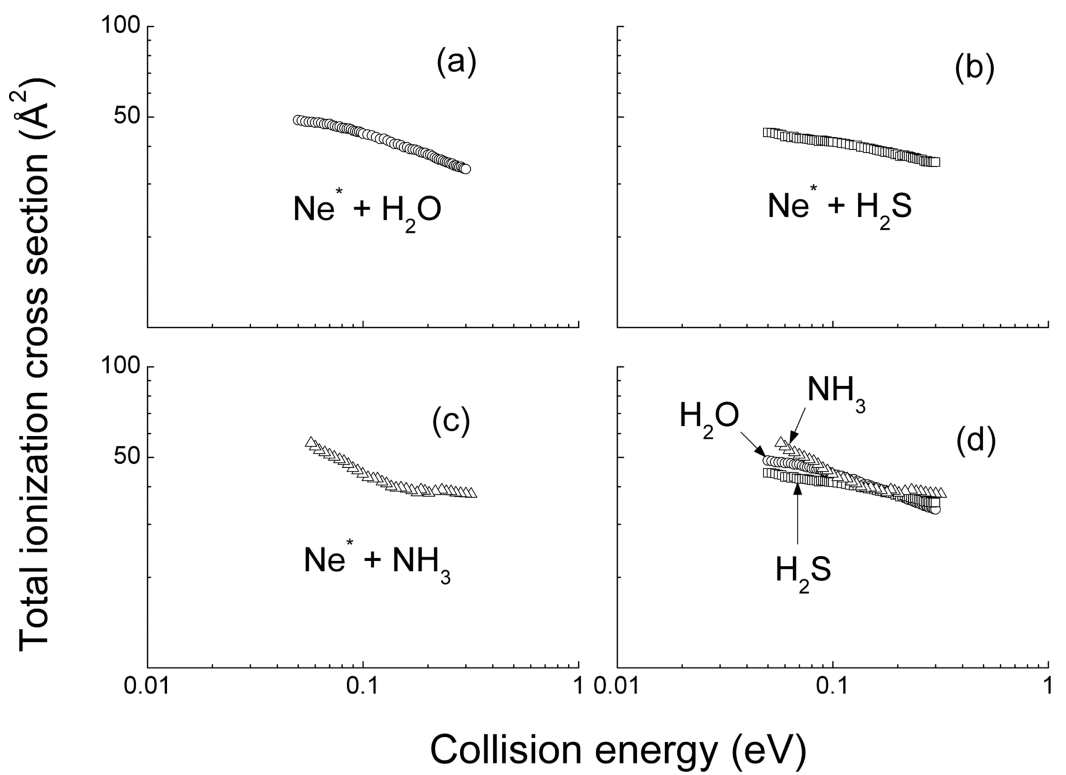

Figure 2. Experimental absolute total ionization cross section for (a) $\mathrm{Ne}^{*}+\mathrm{H}_{2} \mathrm{O}$, (b) $\mathrm{Ne}^{*}+\mathrm{H}_{2} \mathrm{~S}$, and (c) $\mathrm{Ne}^{*}+\mathrm{NH}_{3}$ systems. (d) The cross sections for the three systems are plotted together in order to compare values and slopes.

determination is of about $6 \%$. Panels a-c of Figure 2 plot the absolute value of total ionization cross sections for the three investigated systems in collision with $\mathrm{Ne} *\left({ }^{3} \mathrm{P}_{0,2}\right)$, measured as a function of the collision energy. Moreover, panel $\mathrm{d}$ of the same figure compares cross sections in the same scale in order to emphasize the similar absolute values and the different energy dependence. Figure 3 provides the branching ratio in the case

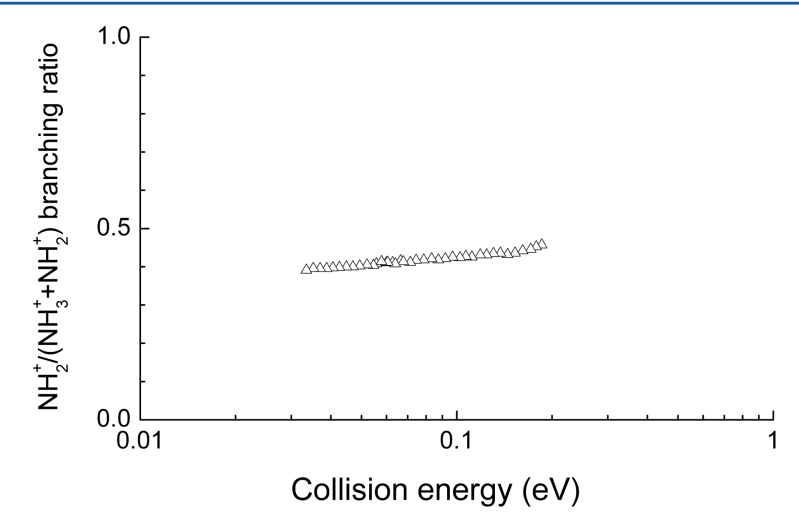

Figure 3. Ratio between the $\mathrm{NH}_{2}{ }^{+}$ion intensity and the total ion products is plotted as a function of the $\mathrm{Ne}^{*}-\mathrm{NH}_{3}$ collision energy.

of $\mathrm{Ne}^{*}-\mathrm{NH}_{3}$ collisions, measured as a function of the collision energy in the same range and defined as a ratio between the mass spectrometric signal of $\mathrm{NH}_{2}{ }^{+}$ions and that of the total ion products. In addition, Table 2 reports for all investigated systems the mass spectrometric determination of branching ratios for ion produced in the main ionization reaction channels, as obtained at an averaged collision energy of 70 $\mathrm{meV}$.

The cross section slopes, different for the three investigated systems and emphasized in panel $\mathrm{d}$ of Figure 2 suggest that such experimental findings can provide additional crucial information on the different angular dependence of the involved total interaction. Considering that the real part has been fully characterized in previous papers, ${ }^{17,18,20}$ exploiting an analytical formulation of the involved interaction components
Table 2. Branching Ratios for Autoionizing Collision of $\mathrm{He}^{*}$ and $\mathrm{Ne}^{*}$ with Hydrogenated Molecules Measured at a Relative Collision Energy of $70 \mathrm{meV}$

\begin{tabular}{cccc} 
target species & product ion & $\mathrm{He}^{*}\left(2^{1} \mathrm{~S}_{0}, 2^{3} \mathrm{~S}_{1}\right)$ & $\mathrm{Ne} *\left({ }^{3} \mathrm{P}_{0,2}\right)$ \\
& $\mathrm{H}_{2} \mathrm{O}^{+}$ & $0.773 \pm 10 \%$ & $0.962 \pm 10 \%$ \\
$\mathrm{H}_{2} \mathrm{O}$ & $\mathrm{OH}^{+}$ & $0.189 \pm 10 \%$ & $0.032 \pm 15 \%$ \\
& $\mathrm{O}^{+}$ & $0.038 \pm 15 \%$ & $0.006 \pm 15 \%$ \\
$\mathrm{H}_{2} \mathrm{~S}$ & $\mathrm{H}_{2} \mathrm{~S}^{+}$ & & 1.00 \\
$\mathrm{NH}_{3}$ & $\mathrm{NH}_{3}{ }^{+}$ & & $0.581 \pm 10 \%$ \\
& $\mathrm{NH}_{2}{ }^{+}$ & & $0.419 \pm 10 \%$ \\
\hline
\end{tabular}

and probed analyzing the peak shifts in the Penning ionization electron spectra for the three systems under study, the present data can be relevant to extract for the first time the radial and angular dependence of the imaginary component. In order to demonstrate such a possibility, Figure 4 reports for $\mathrm{Ne}^{*}-\mathrm{H}_{2} \mathrm{O}$ system the comparison of the measured cross section with a semiclassical calculation carried out using the isotropic component for both parts of the optical potential. For the imaginary part, as in previous studies, ${ }^{17,18,20}$ a simple radial exponential function has been adopted, while the real part has been evaluated averaging the corresponding potential energy surface considering the six basic directions that coincide with the three Cartesian axes. Moreover, a more accurate average has been used by considering a 32-point grid properly distributed in the sphere. The calculations provide cross sections in the right scale of the absolute value but they are unable to reproduce the correct energy dependence.

For the $\mathrm{Ne}^{*}-\mathrm{H}_{2} \mathrm{O}$ system, the total ionization cross sections, as a function of the collision energy, are reported in Figure 5 where the experimental results are compared with calculations performed by assuming that the autoionization events occur exclusively along the six basic directions. Note that this corresponds to assume a fixed mutual orientation of the system during the collision. In other words, such a comparison indicates that an analysis in terms of simple radial interaction components appears to be unable to reproduce the correct slope of measured cross section and the calculated results 


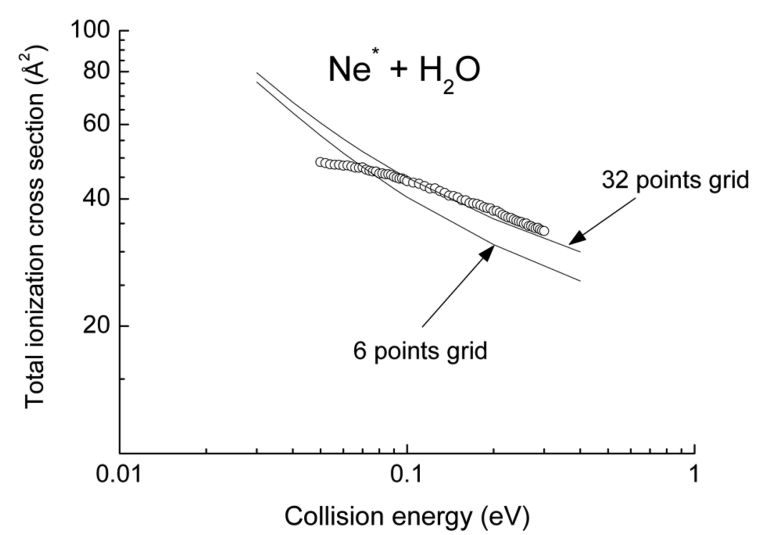

Figure 4. Total ionization cross sections for the $\mathrm{Ne}^{*}-\mathrm{H}_{2} \mathrm{O}$ collision are compared with the calculation performed by a spherical average of the potential energy surface describing the intermolecular interaction. For the spherical average, one grid of 6 and one grid of 32 directions has been adopted. The 6 directions are the basic ones that coincide with those of the three Cartesian axes, whereas the 32 directions are a point grid properly distributed on the sphere. The calculations provide cross sections in the right scale of the absolute value but they are unable to reproduce the correct energy dependence.

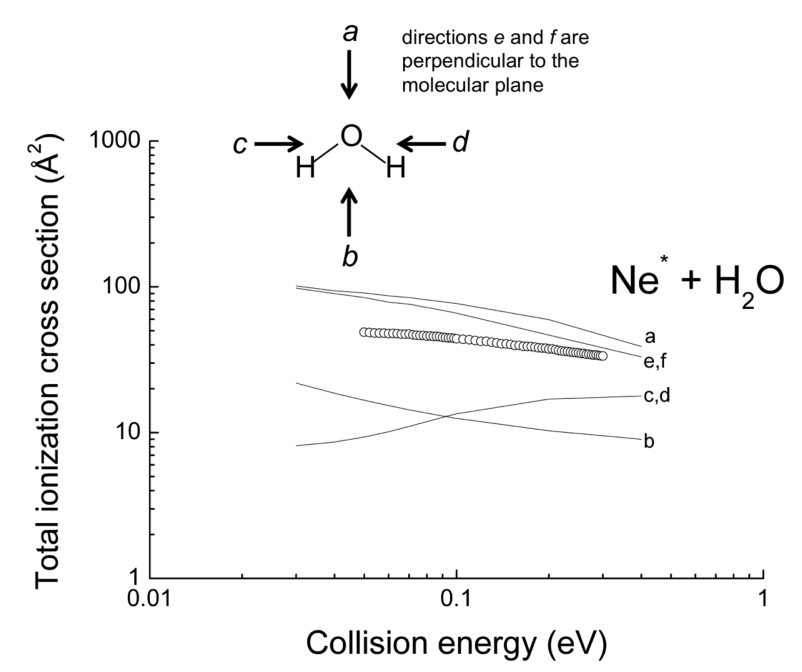

Figure 5. Total ionization cross sections for the $\mathrm{Ne}^{*}-\mathrm{H}_{2} \mathrm{O}$ system compared with calculations performed by assuming that the autoionization events occur exclusively along the six basic directions. Note that this corresponds to the assumption of a fixed mutual orientation of the system during the collision. In other words, this comparison indicates that an analysis in terms of simple radial interaction components appears to be unable to reproduce the correct slope of measured cross section and the calculated results suggest a strong dependence of the reaction on the configuration of the collision complex.

suggest a strong dependence of the reaction on the configuration of the collision complex.

All these experiment findings, which include specifically total ionization values, their energy dependence, and the branching ratios, cast light on the importance of the angular dependence of both real and imaginary interaction components in the selectivity of the autoionization reaction dynamics. Such an analysis is in progress with the objective of reproducing all the available experimental findings with a method fully internally consistent. The characterization of the angular dependence of the imaginary part will be crucial to define with increased accuracy and with respect to previous estimates ${ }^{17,18,20}$ the angular cones that selectively drive the reaction along specific entrance and exit channels.

\section{CONCLUSIONS}

This work provides further experimental information on the stereodynamics of chemi-ionization reactions of water, hydrogen sulfide, and ammonia, which are of great relevance for modeling phenomena occurring in planetary atmospheres and interstellar environments. Experimental observables, presented and discussed in this paper, concern the absolute total ionization cross sections, measured as a function of the collision energy in the thermal range, and the branching ratio for product ions. All these observables have been obtained under the same experimental conditions and their combined analysis will provide important further information on the stereodynamics of these processes. For instance, the $\mathrm{NH}_{2}{ }^{+} /$ $\left(\mathrm{NH}_{3}{ }^{+}+\mathrm{NH}_{2}{ }^{+}\right)$ion ratio appears to be strongly dependent on the ionization involving different molecular orbitals of $\mathrm{NH}_{3}$ that while exhibiting a different spatial orientation selectively involve different configurations of the collision complex.

It has been stressed that chemi-ionization processes are driven by an optical potential, depending on the combination of a real and an imaginary component. The real part has been recently fully characterized for the three systems of interest by exploiting an analytical formulation of the intermolecular interaction tested on the peak structure of the Penning ionization electron spectra. ${ }^{17,18,20,26,27}$ The combined analysis of the experimental observables here presented should allow a characterization of strength, radial, and angular dependence of the imaginary term. Such an analysis is presently in progress in our laboratory and we hope to obtain significant new results in a near future. We should expect to better characterize also the angular cones that define the stereospecificity for the formation of various product ions.

\section{AUTHOR INFORMATION}

\section{Corresponding Author}

*E-mail: stefano.falcinelli@unipg.it. Tel: +39-075-585-3856. Fax: +39-075-585-3864

\section{Notes}

The authors declare no competing financial interest.

\section{ACKNOWLEDGMENTS}

Financial contributions from the MIUR (Ministero dell'Istruzione, dell'Università e della Ricerca) through PRIN 2009 (Grant 2009W2W4YF_002) and PRIN 2010-2011 (Grant 2010ERFKXL 002) projects are gratefully acknowledged. The authors also gratefully thank "Fondazione Cassa di Risparmio di Perugia” for a partial support (Project codes: 2014.0255.021).

\section{REFERENCES}

(1) Benz, A.; Morgner, H. Transition State Spectroscopy with Electrons: The Reaction of $\mathrm{He}\left(2^{3} S, 2^{1} S\right)$ with $\mathrm{Cl}_{2}$. Mol. Phys. 1986, 57, 319-336.

(2) Penning, F. M. Über Ionisation Durch Metastabile Atome. Naturwissenschaften 1927, 15, 818-818.

(3) Atkinson, R.; Baulch, D. L.; Cox, R. A.; Hampson, R. F.; Kerr, J. A., Jr.; Troe, J. Evaluated Kinetic and Photochemical Data for Atmospheric Chemistry: Supplement IV. IUPAC Subcommittee on Kinetic Data Evaluation for Atmospheric Chemistry. J. Phys. Chem. Ref. Data 1992, 21, 1125-1499.

(4) Biondini, F.; Brunetti, B. G.; Candori, P.; De Angelis, F.; Falcinelli, S.; Tarantelli, F.; Teixidor, M. M.; Pirani, F.; Vecchiocattivi, 
F. Penning Ionization of $\mathrm{N}_{2} \mathrm{O}$ Molecules by $\mathrm{He}^{*}\left(2^{3,1} \mathrm{~S}\right)$ and $\mathrm{Ne}^{*}\left({ }^{3} \mathrm{P}_{2,0}\right)$ Metastable Atoms: A Crossed Beam Study. J. Chem. Phys. 2005, 122, 164307.

(5) Brunetti, B. G.; Candori, P.; Cappelletti, D.; Falcinelli, S.; Pirani, F.; Stranges, D.; Vecchiocattivi, F. Penning Ionization Electron Spectroscopy of Water Molecules by Metastable Neon Atoms. Chem. Phys. Lett. 2012, 539-540, 19-23.

(6) Balucani, N.; Bartocci, A.; Brunetti, B. G.; Candori, P.; Falcinelli, S.; Palazzetti, F.; Pirani, F.; Vecchiocattivi, F. Collisional Autoionization Dynamics of $\mathrm{Ne}^{*}\left({ }^{3} \mathrm{P}_{2,0}\right)-\mathrm{H}_{2} \mathrm{O}$. Chem. Phys. Lett. 2012, 546, 34-39.

(7) Alagia, M.; Balucani, N.; Candori, P.; Falcinelli, S.; Pirani, F.; Richter, R.; Rosi, M.; Stranges, S.; Vecchiocattivi, F. Production of Ions at High Energy and Its Role in Extraterrestrial Environments. Rend. Fis. Acc. Lincei 2013, 24, 53-65.

(8) Falcinelli, S.; Pirani, F.; Vecchiocattivi, F. The Possible Role of Penning Ionization Processes in Planetary Atmospheres. Atmosphere 2015, 6, 299-317.

(9) Biondini, F.; Brunetti, B. G.; Candori, P.; De Angelis, F.; Falcinelli, S.; Tarantelli, F.; Pirani, F.; Vecchiocattivi, F. Penning Ionization of $\mathrm{N}_{2} \mathrm{O}$ Molecules by $\mathrm{He}^{*}\left(2^{3,1} \mathrm{~S}\right)$ and $\mathrm{Ne}^{*}\left({ }^{3} \mathrm{P}_{2,0}\right)$ Metastable Atoms: Theoretical Considerations About the Intermolecular Interactions. J. Chem. Phys. 2005, 122, 164308.

(10) Indriolo, N.; Hobbs, L. M.; Hinkle, K. H.; McCall, B. J. Interstellar Metastable Helium Absorption As a Probe of the CosmicRay Ionization Rate. Astrophys. J. 2009, 703, 2131-2137.

(11) Waldrop, L. S.; Kerr, R. B.; González, S. A.; Sulzer, M. P.; Noto, J.; Kamalabadi, F. Generation of Metastable Helium and $1083 \mathrm{~nm}$ Emission in the Upper Thermosphere. J. Geophys. Res. 2005, 110, A08304.

(12) Bishop, J.; Link, R. $\mathrm{He}\left(2^{3} S\right)$ Densities in the Upper Thermosphere: Updates in Modeling Capabilities and Comparison with Midlatitude Observations. J. Geophys. Res. 1999, 104, 1715717172.

(13) Siska, P. E. Molecular-Beam Studies of Penning Ionization. Rev. Mod. Phys. 1993, 65, 337-412.

(14) Brunetti, B. G.; Vecchiocattivi, F. Autoionization Dynamics of Collisional Complexes. In Ion Clusters; Ng, C., Baer, T., Powis, I., Eds.; Springer: New York, 1993; pp 359-445.

(15) Karkoschka, E. Methane, Ammonia, and Temperature Measurements of the Jovian Planets and Titan from CCD-Spectrophotometry. Icarus 1998, 133, 134-146.

(16) Cappelletti, D.; Bartocci, A.; Grandinetti, F.; Falcinelli, S.; Belpassi, L.; Tarantelli, F.; Pirani, F. Experimental Evidence of Chemical Components in the Bonding of Helium and Neon with Neutral Molecules. Chem. - Eur. J. 2015, 21, 6234-6240.

(17) Falcinelli, S.; Bartocci, A.; Cavalli, S.; Pirani, F.; Vecchiocattivi, F. Stereo-Dynamics in Collisional Autoionization of Water, Ammonia, and Hydrogen Sulfide with Metastable Rare Gas Atoms: Competition Between Intermolecular Halogen and Hydrogen Bonds. Chem. - Eur. J. 2016, 22, 764-771.

(18) Falcinelli, S.; Bartocci, A.; Cavalli, S.; Pirani, F.; Vecchiocattivi, F. The Stereo-Dynamics of Collisional Autoionization of Ammonia by Helium and Neon Metastable Excited Atoms Through Molecular Beam Experiments. J. Chem. Phys. 2015, 143, 164306.

(19) Falcinelli, S.; Candori, P.; Bettoni, M.; Pirani, F.; Vecchiocattivi, F. Penning Ionization Electron Spectroscopy of Hydrogen Sulfide by Metastable Helium and Neon atoms. J. Phys. Chem. A 2014, 118, 6501-6506.

(20) Falcinelli, S.; Bartocci, A.; Candori, P.; Pirani, F.; Vecchiocattivi, F. Intermolecular Potential Energy Surfaces for the Interaction Between $\mathrm{H}_{2} \mathrm{X}(\mathrm{X}=\mathrm{O}, \mathrm{S})$ and Metastable $\mathrm{Ne}^{*}\left({ }^{3} \mathrm{P}_{2,0}\right)$ Atom. Chem. Phys. Lett. 2014, 614, 171-175.

(21) Brunetti, B.; Candori, P.; Falcinelli, S.; Lescop, B.; Liuti, G.; Pirani, F.; Vecchiocattivi, F. Energy Dependence of the Penning Ionization Electron Spectrum of $\mathrm{Ne}^{*}\left({ }^{3} \mathrm{P}_{2,0}\right)+\mathrm{Kr}$. Eur. Phys. J. D 2006, $38,21-27$.

(22) Brunetti, B.; Falcinelli, S.; Sassara, A.; de Andres, J.; Vecchiocattivi, F. Auto-Ionization of the Collisional Complexes of
Metastable Neon and $\mathrm{H}_{2}, \mathrm{D}_{2}$, or HD. Chem. Phys. 1996, 209, 205216

(23) Brunetti, B. G.; Candori, P.; Ferramosche, R.; Falcinelli, S.; Vecchiocattivi, F.; Sassara, A.; Chergui, M. Penning Ionization of $\mathrm{C}_{60}$ Molecules. Chem. Phys. Lett. 1998, 294, 584-592.

(24) West, W. P.; Cook, T. B.; Dunning, F. B.; Rundel, R. D.; Stebbings, R. F. Chemiionization Involving Rare Gas Metastable Atoms. J. Chem. Phys. 1975, 63, 1237-1242.

(25) Brunetti, B.; Candori, P.; Falcinelli, S.; Vecchiocattivi, F.; Sassara, A.; Chergui, M. Dynamics of the Penning Ionization of Fullerene Molecules by Metastable Neon Atoms. J. Phys. Chem. A 2000, 104, 5942-5945.

(26) Brunetti, B. G.; Candori, P.; Falcinelli, S.; Pirani, F.; Vecchiocattivi, F. The Stereodynamics of the Penning Ionization of Water by Metastable Neon Atoms. J. Chem. Phys. 2013, 139, 164305.

(27) Arfa, M. B.; Lescop, B.; Cherid, M.; Brunetti, B.; Candori, P.; Malfatti, D.; Falcinelli, S.; Vecchiocattivi, F. Ionization of Ammonia Molecules by Collision with Metastable Neon Atoms. Chem. Phys. Lett. 1999, 308, 71-77. 